\title{
Tra Omero e Melville: Vinicio Capossela sulle tracce di Dante
}

\author{
Francesco Ciabattoni \\ Georgetown University \\ fc237@georgetown.edu \\ https://orcid.org/0000-0002-4350-9929
}

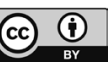

\section{Riassunto}

Questo saggio indaga i riferimenti letterari e specificamente danteschi nei testi delle canzoni del cantautore italiano Vinicio Capossela. Il saggio prende in considerazione alcuni romanzi scritti dal cantautore e i testi delle sue canzoni, in particolare l'album Marinai, profeti e balene del 20II. Attraverso una ricostruzione dell'interesse letterario di Capossela per Moby Dick di Herman Melville e Odissea di Omero, il saggio prende spunto dal suggerimento di Piero Boitani che Melville potrebbe essere stata una lente importante attraverso la quale Jorge Luis Borges lesse Dante. Capossela sembra aver seguito lo stesso percorso letterario, arrivando a Dante proprio attraverso Borges, Melville e Omero.

Parole chiave: Dante; pop; folk; musica; canzone; cantautore; Vinicio Capossela; Melville; Omero; Borges; Boitani; intertestualità.

\begin{abstract}
This essay investigates the literary and specifically Dantean references in the song lyrics of Italian singer-songwriter Vinicio Capossela. The essay takes into consideration some novels written by the cantautore as well as his song lyrics, especially the 20II album Marinai, profeti e balene. Through a reconstruction of Capossela's literary interest in Herman Melville's Moby Dick and Homer's Odyssey, the essay takes a suggestion from Piero Boitani that Melville might have been an important lens through which Jorge Luis Borges read Dante. Capossela seems to have followed the same literary path, arriving to Dante precisely through Borges, Melville and Homer.
\end{abstract}

Key Words: Dante; pop; folk; music; song; singer-songwriter; Vinicio Capossela; Melville; Homer; Borges; Boitani; intertextuality. 
"Se poi ci volgiamo all'immaginario ulissiaco, vedremo che esso è profondamente intrecciato nel tessuto di Moby-Dick ... Achab è un Ulisse ultradantesco: un vecchio che abbandona moglie e figlio, che convince i suoi uomini a seguirlo nell'impresa disperata, che pronuncia un' 'orazion', la quale si trasforma subito in cerimonia di iniziazione e in atto di comunione diabolica." (Boitani 2003:33-34)

$\mathrm{D}$ i Vinicio Capossela è lecito parlare su una rivista dedicata a Dante non solo per il riconoscimento che la Società Dante Alighieri assegnò attraverso i Circoli di lettura italiani ed esteri al suo Il paese dei coppoloni (Feltrinelli 20I5) come miglior libro nell'ambito della rassegna "La Dante al Premio Strega”, ma anche per i meriti specificamente letterari di quel libro e soprattutto di alcuni suoi testi per canzone. Nato ad Hannover nel 1965, Capossela è cresciuto tra Reggio Emilia i paesi dell'Irpinia (Calitri e Andretta) originari dei genitori, dove ogni anno il cantautore torna per dirigere lo Sponz Fest, il festival di arte, musica e cultura non solo popolare. ${ }^{I}$ Il presente saggio, che termina con la trascrizione di un'intervista rilasciatami dal cantautore di fronte al pubblico americano alla Georgetown University di Washington DC il 4 maggio 2019, propone un commento dell'interesse letterario e specificamente dantesco del cantautore.

Andiamo per ordine e ripercorriamo la strada che ha portato Vinicio $\mathrm{Ca}-$ possela, autore anche di quattro romanzi, a interessarsi a Dante negli ultimi anni. ${ }^{2}$ Il suo interesse per la contaminazione di canzone e letteratura è antico e s'intreccia all'interesse per le tradizioni popolari, in uno spirito non dissimile-anche se con stile e metodo assai diversi, perché diversi sono il contesto e i mezzi scelti per la rappresentazione artistica-da quello che spinse Pasolini a ricercare il sacro e l'epico nella cultura contadina, o dallo spirito che dimostrava Francesco De Gregori mentre nel 2005 cantava un pot-pourri dantesco da Inferno e Purgatorio sulle note, suonate da Ambrogio Sparagna, di una pizzica

I. Si ringrazia Alessandro Gambino, dell'ufficio stampa e comunicazione di Capossela, che in una comunicazione privata così definisce il significato della parola "sponz": "viene da 'sponzare', una parola vicino a 'sponsale' (da qui l'origine dello Sponz Fest, nato non come una festival ma come una festa di comunità per il piacere di stare insieme e recuperare le tradizioni legate al rito più ancestrale: lo sposalizio). Il termine sponzare in realtà in molti paesi del sud Italia viene da sponza, cioè spugna. Sponzare e sponzarsi dunque letteralmente significa imbeversi, inzupparsi, rendersi fradici... Normalmente si dice del baccalà, unico pesce che un tempo raggiungeva i paesi dell'interno, che viene venduto rigido e salato e per rendersi commestibile deve essere messo in ammollo, deve appunto "sponzare" almeno tre giorni. A quel punto perde rigidità e salinità e diventa buono da mangiare. Così vuole fare lo Sponz Fest: ammollarsi, infradiciarsi di musica e racconti, farci partecipare a un travolgente rito dionisiaco in cui si balla fino a cadere 'sponzati come baccalà, darsi l'occasione di riflettere sul senso della comunità e su un modello di relazionamento sociale ed economico.” La medesima definizione appare anche sul sito http://www.campaniaslow.it/20I5/08/22/dalunedi-lo-sponz-fest-ma-che-significa-sponzare/ in un articolo a firma di Carlo Scatozza.

2. Non si muore tutte le mattine, Feltrinelli 2004; In clandestinità (con Vincenzo Costantino), Feltrinelli 2009; Tefteri, Il Saggiatore 2013 e Il paese dei coppoloni, Feltrinelli, 20I5. 
salentina a Melpignano nella serata conclusiva de "La Notte della Taranta" (Cotugno \& Gargano 20I6:I26-I28). Vi partecipò anche Capossela nel 2008 con un pezzo trascinante: "Il ballo di San Vito", (1996) che descrive gli effetti di una malattia batterica, la còrea di Sydenham, volgarmente detta appunto "ballo di san Vito", i cui sintomi, simili al tarantismo, servono a Capossela per suggerire un approccio non-razionalista alla conoscenza, consentito proprio dalla possessione della malattia ("Ho il ballo di San Vito e non mi passa / il cerusico ha gli occhi ribaltati ed il curato non se ne cura / il ragioniere non ragiona / santo Paolo non perdona"). Sfruttando assonanze, paronomasie e calembour, "Il ballo di San Vito" sprigiona una forza musicale irresistibile, contagiosa come il morso della tarantola, rivisitando una tradizione folklorica millenaria, in sintonia con quanto fece poi Eugenio Bennato nel 1999 con $\mathrm{Ta}$ ranta Power, sull'onda di un nuovo revival della musica popolare. Quest'ultima, dunque, è una delle due componenti principali dell' ispirazione artistica di Vinicio Capossela, il quale mescola, ibrida e contamina volentieri il folk con il letterario. Le suggestioni letterarie in Vinicio Capossela sono ampie e numerose, e presenti già da questi suoi primi successi, per esempio si potrebbe sospettare (anche con maggiore convinzione di quanto fa Elisabetta Cucco (2009: 42-48) che "Pioggia di novembre" (Il ballo di San Vito, 1996: "nuvole non ne vedo / di qua è una striscia di cielo.../ piove sulle urla dei villani / sul cimitero monumentale") porti echi montaliani e una sostanziale affinità di struttura con "Piove" (Satura II, 1971: 345-347: "Piove / da un cielo che non ha / nuvole... / piove / sui cipressi malati /del cimitero"); qualche anno più tardi il cantautore metteva in musica, interpretandoli, tre sonetti di Michelangelo ("Non posso altra figura imaginarmi", "Fuggite, amanti, Amor, fuggite 'l foco" e "Ogni van chiuso, ogni coperto loco") nell'album Renaissance (Naïve 200I) del musicista francese Philippe Eidel; quindi riecheggiava il Coleridge della "Ballata del vecchio marinaio" ("Santissima dei Naufragati”, Ovunque proteggi 2006) e mescolava frammenti dal Nuovo Testamento ("Non sappia la tua destra che fa la tua sinistra") con citazioni dai Salmi ("nel sangue dell'empio mi laverò i piedi", dal Salmo 58) in "Non trattare" (Ovunque Proteggi 2006). Inoltre, tutto l'album più recente, Ballate per nomini e bestie (2019), è un vero e proprio turbinio di riferimenti letterari, un ballo-riprendo una metafora del cantautore stesso, che d'altronde vi include "Danza macabra", la cui ispirazione è "Ad mortem festinamus" del Llibre Vermell de Monserrat - al quale prendono parte diversi autori della letteratura mondiale: Antonin Artaud ("La peste"); Oscar Wilde del quale Capossela riscrive "The Ballad of Reading Gaol" in "La ballata del carcere di Reading"; John Keats ("La Belle Dame Sans Merci"); Francesco d'Assisi ("Perfetta Letizia" riprende una delle pagine più note dei Fioretti); Richard de Fournival, il cui Bestiario d'amore popola di creature fantastiche versi e prose di Capossela. L'album racconta l'animalità dell'uomo ("Le loup 
garou") e l'umanità delle bestie ("La giraffa di Imola") e la ricerca del mito ("Nuove tentazioni di Sant'Antonio" accosta il santo a un novello prometeo), muovendosi ancora una volta fra tradizione popolare e letteratura. I frequenti riferimenti alla condizione dei migranti fungono da elemento attualizzante, alludendo, in "Il povero Cristo", a Christ in Concrete di Pietro Di Donato.

Sulla stessa lunghezza d'onda, quella cioè del folk revival, mischiato al riuso letterario, si collocano anche Le canzoni della Cupa (2016), che recupera la dimensione mitica della terra con l'impiego di vocalità dure e sonorità tipiche della musica popolare, tamburi, organetti, fisarmoniche. Un album doppio che sta a metà fra I racconti dell'Ohio di Sherwood Anderson e la mitologia contadina dell'Irpinia, ma che diventa espressione di un sud universale, un pensiero meridiano memoriale-onirico (in Il paese dei coppoloni "il tempo dell'accidia meridiana" è anche "il tempo della memoria e del sogno" Capossela 2004: 252) e popolare che passa anche da Carlo Levi e Matteo Salvatore. Il disco di Capossela si divide in due parti: "polvere" e "ombra", che evoca l'oraziano "pulvis et umbra sumus" (Odi IV.7.I6, Nuzzo e Gianotti 2009: 296), espressione della caducità dell'uomo, nell'avvicendarsi delle stagioni, probabilmente non senza l'ombra di Chiedi alla polvere di John Fante, autore caro a Capossela, poiché "L'accolita dei rancorosi" (Il ballo di San Vito, 1996) si ispira a La confraternita dell'uva, per la cui edizione einaudiana del 2004 il cantautore firmò un'introduzione (si veda Fiore 2006).

Anche la prosa dei romanzi di Capossela fa frequente ricorso all'attualizzazione di motivi cristiani, alla contaminazione dell'antico con il moderno, alla rivisitazione e al riadattamento del mito e del classico letterario in un contesto contemporaneo e preferibilmente "paesano." Nei suoi libri la struttura narrativa appare volontariamente frammentata, in uno sforzo di sollecitare la risposta emotiva del lettore: "Vorrei che queste pagine si potessero prendere a etto, sfuse, a capitoli, a ognuno la parte che gli serve, come dal macellaio" (Capossela 2004:6) e la ricerca di conoscenza appare fondata sul fattore emotivo più che sulla cognitività razionale, come si legge in Non si muore tutte le mattine, primo romanzo del cantautore: "preferisco rimanere un'impressione, preferisco le impressioni. Le impressioni emozionano. È inutile conoscere: molto meglio supporre" (Capossela 2004: 252). La conoscenza, e i mezzi per acquisirlaaspetti di un'epistemologia che interessarono profondamente Dante-, divengono tematiche centrali in $\mathrm{Il}$ paese dei coppoloni, nel quale al recupero del sacro e del mito è affidato il compito, altissimo, di riportare umanità e ritualità in un mondo che ha perso tali dimensioni. Ecco allora che affiorano fra le righe alcune suggestioni dantesche: quando l'anonimo protagonista di Il paese dei coppoloni, alla ricerca per tutto il romanzo di qualcosa di indefinito ma vitale, si reca all'oracolo della Totara, il volto e la voce di questa Pizia del meridione 
si manifestano con modalità simili a quelle con cui la voce di San Tommaso arriva alla mente di Dante nel cielo del sole:

... come i cerchi nell'acqua di stagno si formano, dopo che una pietra caduta gli scuote il ventre d'abisso, così attorno a quei nodi l'albero s'increspò in cerchi che fecero ridente la bocca e gli occhi vispi comparvero. (Capossela 2015: 37)

Il passo presenta qualche affinità con l'incipit del quattordicesimo canto del Paradiso:

Dal centro al cerchio, e sì dal cerchio al centro

movesi l'acqua in un ritondo vaso, secondo ch'è percosso fuori o dentro:

ne la mia mente fé sùbito caso questo ch'io dico, sì come si tacque la glorïosa vita di Tommaso

(Paradiso XIV, I-6)

Si trovano echi danteschi di varia rilevanza in tutto il romanzo, talvolta commisti a disquisizioni dal carattere popolaresco su "Nfierno" e Paradiso, e sui peccati capitali di ira, lussuria, gola e "'mmiria", cioè invidia (Capossela 20I5: 233, 253).

Tornando alle canzoni, non ci sorprenderemo più, ormai, che i classici della letteratura trovino luogo nelle opere di artisti testi folk, rock, pop o come si voglia definirli, se Richard F. Thomas (2017) ha ravvisato in Bob Dylan un pantheon di poeti classici e se già quarant'anni fa Julia Kristeva (I980: 36) aveva compreso che ogni testo è un crocevia di altri testi nei quali "several utterances, taken from other texts, intersect and neutralize each other." I cantautori, italiani e non, scrivono in una completa libertà di ispirazione, il che non li vincola ad alcuna tradizione, men che meno a quella letteraria, eppure talvolta si avvalgono di tecniche di scrittura che sono strettamente letterarie, prelevando, ritagliando, riecheggiando e citando in maniera significativa. Giorgio Pasquali nel suo famoso saggio "Arte allusiva" circoscriveva alla poesia colta questo fenomeno:

In poesia culta, dotta io ricerco quelle che da qualche anno in qua non chiamo più reminiscenze, ma allusioni, e volentieri direi evocazioni e in certi casi citazioni. Le reminiscenze possono essere inconsapevoli; le imitazioni, il poeta può desiderare che sfuggano al pubblico; le allusioni non producono l'effetto voluto se non su un lettore che si ricordi chiaramente del testo cui si riferiscono (Pasquali 1952:II).

Fenomeno che si può tuttavia osservare anche nella scrittura di certe canzoni, come ho mostrato per i casi di Vecchioni, Guccini, Branduardi, De Gregori, De André e Baglioni (Ciabattoni 2016). È possibilissimo che non tutti questi cantautori, al momento di scrivere le canzoni nelle quali impiegano 
riferimenti letterari, fossero pienamente consapevoli della letterarietà dell'operazione che eseguivano, ma ciò rende forse ancora più significativo il loro ricorso alla citazione, all'allusione, o le loro reminiscenze letterarie, perché in tal caso queste reminiscenze, allusioni e citazioni sarebbero il sintomo di qualcosa di profondo, dell'amore per la letteratura, della necessità spontanea di adottare un codice espressivo. E se altri cantautori prima di Capossela si sono riferiti a Dante, negli anni '7o iconoclasticamente contro il capitale culturale nel senso definito da Pierre Bourdieu (1986) - per muovere una critica all'obsoleto sistema scolastico italiano (Venditti, Vecchioni) o per colpire l'ipocrisia della classe borghese (De André) —in tempi più recenti Angelo Branduardi, Francesco De Gregori, Niccolò Agliardi e Caparezza hanno tratto ispirazione senza vis polemica o sfumature politiche (Ciabattoni 20I7). Del poeta della Commedia interessa qui l'aspetto di poeta, profetico e di cultore del mito. Infatti, se Dante "retells customary mythic events in a vernacular tongue and from a more personal point of view" (Meisel 20I0: 22), comprendiamo allora l'interesse di Vinicio Capossela, che tanto nelle canzoni quanto nei libri mette in moto una ricerca della ritualità, del primordiale, delle radici mitiche della propria cultura al punto di riscrivere interi passi danteschi, come il famoso canto di Ulisse, in certe sue canzoni ("Nostos") o di riecheggiare, altrove e più genericamente, l'universo metafisico della Commedia (ma non senza un po' di spirito boccaccesco e una strizzatina d'occhio a Mark Twain): “... il Giacchettaro che ogni peccato commetteva per essere certo di finire allo 'Nfierno che in Paradiso secondo lui c'erano solo criame e pizoche" (Capossela 2004: 253).

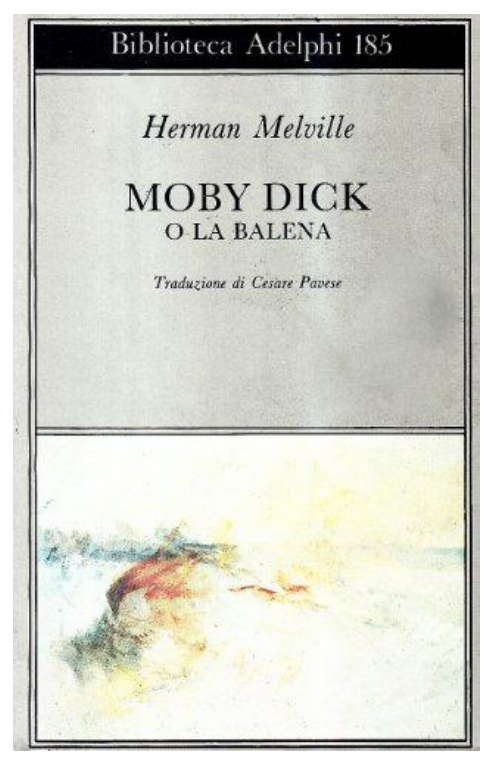


È invece nell'album del 20II, Marinai, profeti e balene, che possiamo trovare una più organica influenza dantesca, che si esprime in rielaborazioni di elementi testuali letterari, allusioni, contaminazioni di motivi, idee e immagini. Due testi soprattutto governano l'ispirazione delle diciannove canzoni di questo doppio LP: da un lato l'Odissea, che fornisce l'ispirazione per i marinai, e dall'altro Moby Dick, che oltre va oltre i marinai e dà conto anche delle balene. "Il grande Leviatano" è una messa in musica di "The Ribs and Terrors in the Whale" di Herman Melville (nella traduzione di Cesare Pavese, Adelphi 194I), un testo che già Melville stesso associava al canto nel IX capitolo di Moby Dick, intitolato "Il sermone", e collegato alla vicenda biblica del profeta Giona. In Melville, infatti, Padre Mapple intona l'inno, poi seguito dalla congrega alla quale si rivolge. E per arringare e dirigere la folla, egli grida — come farebbe l'Ulisse dantesco-ripetutamente: "compagni", ovvero shipmates nell'originale inglese, ma se Capossela ha letto la traduzione di Pavese avrà letto, appunto "compagni", un termine che lo scrittore e traduttore piemontese avrà scelto forse proprio con quei versi di If. XXVI in mente: "Io e' compagni eravam vecchi e tardi" (IO6) e "Li miei compagni fec' io sì aguti" (I2I). Naturalmente il sermone di padre Mapple risulta piuttosto anti-ulissiaco, con le sue ammonizioni e gli inviti alla prudenza, tuttavia qualche reminiscenza dantesca si può scorgere nella menzione del viaggio attraverso Cadice e Gibilterra, e nella descrizione dell'inferno intravisto nella bocca della balena ("Io vidi spalancarsi la bocca dell'Inferno / con pene e con dolori d'orrenda privazione / che, solo chi l'ha provata, sa cos'è in eterno: cadevo nell'abisso della disperazione" Melville 2009:753). L'ispirazione melvilliana pervade pure "L'Oceano Oilalà", "La bianchezza della balena", "Billy Budd" e "I fuochi fatui", mentre "Pryntyl" è tratta da Scandalo negli abissi di Louis-Ferdinand Céline, "Lord Jim" si ispira all'omonimo romanzo conradiano e "Job" si rifa alla figura biblica di Giobbe. La seconda parte del doppio LP è invece dedicata principalmente all'Odissea con titoli come "La lancia del Pelide", "Vinocolo" (in cui il cantautore si narra nei panni di Polifemo giocando sull'assonanza tra vino, monocolo e il proprio nome e l'io narrante- o cantante—si sposta dalla parte dell'"Altro": a dire "io" è Polifemo e Ulisse è invece l' "Altro", il nemico che lo sconfigge), "Aedo", "La Madonna delle Conchiglie", "Calipso", "Dimmi Tiresia", "Nostos" e "Le sirene". Dopo marinai e balene, per spiegare il terzo elemento, cioè i profetinon a caso posto in mezzo ai primi due-, non basterà invocare Tiresia, pure protagonista di una delle canzoni dell'album, o le molte istanze di profezia che pervadono il romanzo di Melville, ma converrà guardare anche a Dante, che si può considerare, almeno nell'ambito specifico della scrittura di questo album, il punto di collegamento fra Odisseo e Achab. Quale può essere la relazione

3. "I saw the opening maw of hell, / With endless pains and sorrows there; / Which none but they that feel can tell / - Oh, I was plunging to despair" (Melville 1993:36) 
fra Melville e Dante? Cosa spinge Capossela a far dialogare fra loro l'Ulisse di Dante, l'Odisseo di Omero e l'Achab di Melville? I legami fra i tre eroi dei mari sono stati spiegati con finezza Piero Boitani in un saggio del 2003, ricostruendo un percorso letterario che passa attraverso la visione di un moderno Omero, l'aedo visionario non vedente Jorge Luis Borges.

\section{Piccola Biblioteca 578}

Jorge Luis Borges

LA MONETA

DI FERRO

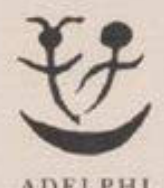

ADELPHI

Il geniale scrittore argentino, i cui Nove saggi danteschi sono piuttosto noti ai lettori italiani, dedicò una poesia anche a Herman Melville, che vi figura come un moderno Dante, anzi come un moderno Ulisse:

\section{Herman Melville}

Siempre lo cercó el mar de sus mayores, los sajones, que al mar dieron el nombre Ruta de la ballena, en que se aúnan las dos enormes cosas, la ballena y los mares que largamente surca. Siempre fue suyo el mar. Cuando sus

vieron en alta mar las grandes aguas ya lo había anhelado y poseído en aquel otro mar, que es la Escritura, o en el dintorno de los arquetipos. Hombre, se dio a los mares del planeta $\mathrm{y}$ a las agotadoras singladuras
Herman Melville

Sempre lo circuì l'oceano dei suoi avi, I Sàssoni, che il mare denominarono Strada della balena, così associando Le due enormi cose, la balena E i mari ch'essa lungamente solca. Sempre fu suo il mare. Quando i suoi [occhi

Videro le infinite acque oceaniche, Già le aveva bramate e possedute In quell'altro mare che è la Scrittura. O nel disegno ideale degli Archètipi. Uomo, si consacrò ai mari del pianeta E alle sfibranti navigazioni, 
y conoció el arpón enrojecido por Leviathán y la rayada arena y el olor de las noches y del alba $y$ el horizonte en que el azar acecha y la felicidad de ser valiente y el gusto, al fin, de divisar a Ítaca. Debelador del mar, pisó la tierra firme que es la raíz de las montañas

$y$ en la que marca un vago derrotero, quieta en el tiempo, una dormida

A la heredada sombra de los huertos, Melville cruza las tardes de New England pero lo habita el mar. Es el oprobio del mutilado capitán del Pequod, el mar indescifrable y las borrascas y la abominación de la blancura. Es el gran libro. Es el azul Proteo.
E conobbe l'arpione insanguinato

$\mathrm{Da}$ Leviathan e la rigata arena

E l'aroma delle notti e dell'alba

E l'orizzonte ove la sorte guata

E la felicità d'aver coraggio

E il gaudio, infine, di scorgere Itaca.

Debellatore del mare, calcò la terra

Ferma ove hanno basamento le

[montagne

E sulla quale un vago rombo traccia,

Quieta nel tempo, un'assopita bussola.

All'ereditata ombra degli orti

Melville passeggia nelle sere del New

Ma in lui dimora il mare. ̀̀ [England

$$
\text { [l'obbrobrio }
$$

Del mutilato capitano del "Pequod", Il mare impenetrabile e gli uragani E l'abominazione della bianchezza. È il grande libro. È l'azzurro proteo. ${ }^{4}$

Boitani (2003: 27), sulla scorta di Lea Bertani Newman, ci informa che Melville acquistò una copia della traduzione inglese della Commedia ad opera di Henry Francis Cary (I772-I844). 5 È noto che diverse opere di Melville presentano risonanze dantesche: Pierre, "The Tartarus of Maids," "The Encantadas" (Newman 305), quindi un legame diretto fra lo scrittore americano e il poeta della Commedia è provato, ma certamente la mediazione di Borges, il quale vedeva Achab come una riproposizione del mito di Ulisse, non andrà sottovalutata, poiché come indica ancora Boitani, "Achab... 'compie' Ulisse cercando di portare all'estrema conclusione l'ultima ribellione della sua prefigurazione, contestando attivamente l'Altrui che ha voluto il naufragio di Ulisse" (Boitani 2003:35-36). A dar prova che anche Capossela ha ripercorso il medesimo cammino letterario con cui Borges porta luce al rapporto Melville-Dante, sono le parole dello stesso cantautore, raccolte da Daniele Sidonio durante una lezione tenuta a Ca' Foscari il I6 Dicembre 20II:

4. J.L. Borges, "Herman Melville" p. 136. Trad. di C. Vian, in J.L. Borges, La moneta di ferro, pp. 57-58.

5. H. F. Cary (Transl.), The Vision: or, Hell, Purgatory, and Paradise of Dante Alighieri. La copia appartenuta a Melville dovrebbe essere tuttora nell'archivio privato di William Reese (New Haven, CT, Newman 305), o almeno lo era nel 1993, data di pubblicazione dell'articolo di Newman. 
[Achab e Ulisse] sono due figure che quasi arrivano a coincidere. Borges, per esempio, nei suoi Saggi danteschi, sostiene che Achab è l'Ulisse di Dante, è l'Ulisse senza il ritorno. Infatti il finale di Moby Dick è simile a quello del canto XXVI [...] Una lotta titanica e dopo, 'come altrui piacque', il mare si richiude. (Sidonio 20I4: I6)

Capossela cita il saggio in cui Borges - chiamando in causa anche Eliot, Tennyson, Andrew Lang e Longfellow_percepisce l'affinità "dell'Ulisse infernale con l' altro sventurato capitano: Achab di Moby Dick. Questi, come quegli, costruisce la propria perdizione a forza di veglie e di coraggio; il tema generale è lo stesso, la conclusione e identica, le ultime parole sono quasi uguali [...] entrambe le finzioni, alla luce di questo prodigioso giudizio, sono il processo di un occulto e intricato suicidio" (Borges 200I: 49).

$\mathrm{Si}$ potrà poi osservare un'eco dantesca anche nella canzone dedicata a Tiresia, figura che sia Dante che Odisseo incontrano nell'aldilà e che già ispirò i Genesis ("The Cinema Show", Selling England by the Pound, 1973). Se per Dante Tiresia era un indovino pagano da collocare nella quarta bolgia accanto a Anfiarao e Michael Scott, per Omero è invece ancora il profeta che rivela informazioni preziose a Odisseo. L'interesse di Capossela in "Dimmi Tiresia" è nel ruolo della conoscenza e dunque qui l'Odisseo cantato in prima persona da Capossela oscilla fra quello omerico, che scende nell'Ade per parlare con Tiresia e poi con la propria madre, e quello dantesco, il cui folle ardore di conoscenza è causa della tragedia che lo inabissa col suo equipaggio. Nell'XI libro dell'Odissea, il laerziade interroga Tiresia su se e come potrà ritornare a Itaca, ma è poi Anticlea, la madre di Odisseo, a bere il sangue che la rende capace di conversare coi vivi e rivelare al figlio notizie di Itaca, Laerte, Telemaco, e soprattutto, Penelope. Nella canzone, Odisseo appare invece torturato da un dubbio più profondo: non solo "Se la donna mia mi aspetta se è fedele" ma anche se sia meglio "Sapere o non sapere" circa la fedeltà della sua sposa. Come intuisce bene Trifone Gargano (2018: 5I) il verso di Capossela "Dimmi Tiresia", che presta poi anche il titolo alla canzone, riprende il passo omerico in cui Odisseo incalza il profeta per ottenere nuove informazioni

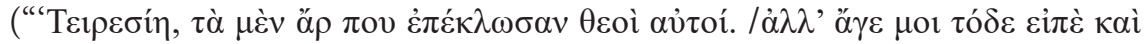

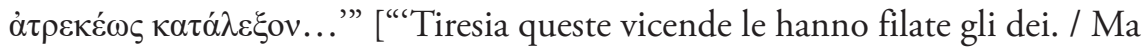
un'altra cosa ora dimmi e parla sincero..."'] Omero, Odissea XI.I39-I40) ma non senza-per il riuso dell'imperativo bisillabo che precede il nome-una certa reminiscenza del "Vedi Tiresia" (If. XX, 40) col quale Virgilio mostra al pellegrino l'ombra del greco, declassato a indovino nell'inferno cristiano, ma riabilitato allo stato profetico da Capossela. E proprio perché profeta, Tiresia può a buon diritto far parte dell'album Marinai, profeti e balene, che il cantautore ha definito una "Marina Commedia", di nuovo lasciandosi attrarre dalla parafrasi del linguaggio dantesco. 


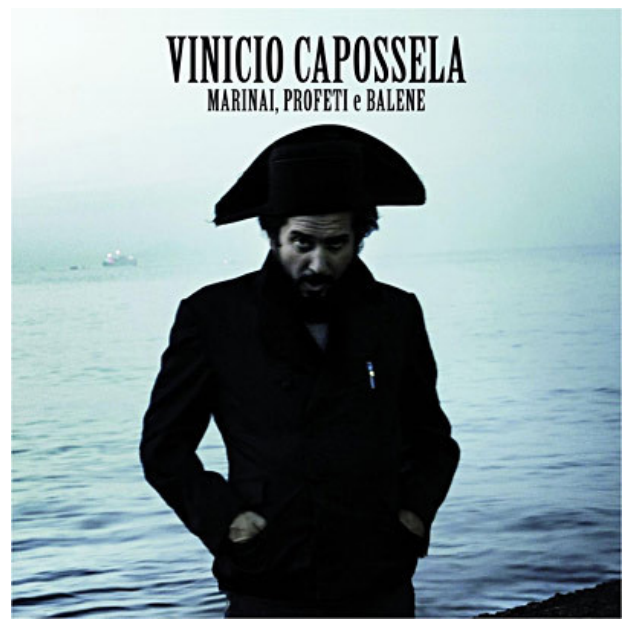

"La lancia del Pelide" applica all'amore la proprietà della mitica arma di Achille, capace di ferire con il primo colpo e di sanare la ferita con il secondo ("Così sei tu, Mia bella tu / Tu che puoi uccidermi e farmi risorgere"). Anche qui sarebbe difficile negare una mediazione dantesca, poiché proprio alla lancia dell'eroe greco il poeta si riferiva nella similitudine del rimbrotto di Virgilio dopo l'incontro con Mastro Adamo e Sinone:

Una medesma lingua pria mi morse, sì che mi tinse l'una e l'altra guancia, e poi la medicina mi riporse; così od' io che solea far la lancia d'Achille e del suo padre esser cagione prima di trista e poi di buona mancia. (If. XXXI, I-6)

Vi è poi la ghost track con cui si chiude il disco, intitolata "Le sirene". Oltre al possibile riferimento a $\mathrm{Pg}$. XIX, I9-2I, essa presenta un verso in cui può leggersi l'orma di Dante: "perché continuare fino a vecchiezza / fino a stare male", sul quale si allunga l'ombra di Ulisse (quello dantesco) e del suo viaggio intrapreso quando lui e i compagni erano "tardi e lenti". Ma è senza dubbio "Nostos" la canzone nella quale i riferimenti danteschi sono più cospicui: la canzone è costruita intrecciando insieme versi del XXVI canto dell'Inferno e versi di Omero, con una tecnica da collage che inserisce fra i versi danteschi parole originali del cantautore che confermano lo stile deliberatamente frammentario di una versificazione che ricerca una via quasi mistica ("oltre il recinto della ragione") alla "canoscenza". Ecco il testo della canzone per intero: 
Né pietà di padre, né tenerezza di figlio, né amore di moglie ma misi me per l'alto mare aperto oltre il recinto della ragione, oltre le colonne che reggono il cielo, fino alle isole fortunate, purgatorio del paradiso nostos nostos fino alle terre retro al sol e sanza gente Itaca ha dato il viaggio le sue ombre di viti nel sole e nel miraggio le abbiam portate dentro come una bussola c'ha fatto andare oltre gli incantesimi, e i Lestrìgoni oltre le lusinghe dell'eterna giovinezza ma a ritornare ora la troveremmo vuota di gente e piena di sonno Itaca ha dato il viaggio, Itaca ha dato il viaggio, l'hai avuta dentro, ma non ci troverai nessuno.

Fatti non foste a viver come bruti, ma per seguire virtute e canoscenza considerate la vostra semenza, considerate la vostra semenza, nostos nostos, perdere il ritorno batti le ali, fare da remi al volo ali al folle volo!

batti le ali, fare da remi al volo ali al folle volo!

fino alle terre retro al sol e sanza gente fino alle terre retro al sol e sanza gente.

Vi spiccano alcuni dei versi più noti del canto di Ulisse, che fungono da tessere per un mosaico nel quale la tensione odeporica è invertita, cioè il punto di attrazione è Itaca, l'obiettivo il ritorno (il nostos, appunto), e alcuni passi della canzone ("Ma a ritornare ora / La troveremmo vuota di gente e piena di sonno") mescolano il "mondo sanza gente" di If. XXVI, II7 con suggestioni provenienti dal primo canto del poema dantesco ("Io non so ben ridir com'i' v'intrai, / tant'era pien di sonno" If. I, IO-II). Si tratta senza dubbio di una lettura libera e personale, a tratti geniale, del mito del viaggio e del ritorno, in cui, come ha dichiarato il cantautore nell'intervista rilasciata alla Georgetown University e riportata al termine di questo saggio, si potrà vedere in filigrana il viaggio di chi migra, di chi si allontana dalla propria Itaca. A Odisseo Capossela si sente vicino, forse pure vi si identifica, se il protagonista di Tefteri può in qualche misura considerarsi affine al narratore: "Allora Odisseo è uguale a me. Senza un porto in cui tornare. Ospite. Apparizioni femminili, simulacri, Solitudine. Ospite" (Capossela 2013: 8I). Le canzoni di Capossela nascono da 
questo processo magmatico, da questa creativa e felice rielaborazione di temi, stilemi e parole provenienti dagli scrittori di cui l'onnivoro cantautore si pasce per la sua propria ricerca delle origini, per la sua riscoperta della ritualità, per riproporre e attualizzare il mito, il senso della conoscenza e l'importanza dell'avventura.

\section{Bibliografia}

Boitani, P. (1992). L'Ombra di Ulisse. Figure di un mito. Bologna: Il Mulino.

Boitani, P. (2003). "Moby Dante" in Studi di letterature comparate in onore di Remo Ceserani, a c. di Mario Domenichelli, Pino Fasano, Mario Lavagetto e Nicola Merola, 2 voll. Manziana, Vecchiarelli, vol. I, 23-42.

Borges, J.L. (1989). Obras completas, 2 voll. Buenos Aires: Emecé.

Borges, J.L. (200I). "Luultimo viaggio di Ulisse" in Nove saggi danteschi. Milano: Adelphi.

Borges, J.L. (2008). La moneta di ferro. Trad. Cesco Vian. Milano: Adelphi.

Bourdieu, P. (1986). "The Forms of Capital" in Handbook of Theory and Research for the Sociology of Education, a c. di J. G. Richardson. Greenwood Press, 24I-258.

Capossela, V. (2004). Non si muore tutte le mattine. Milano: Fetrinelli.

Capossela, V. (2013). Tefteri : il libro dei conti in sospeso. Milano: Il saggiatore.

Capossela, V. (20I5). Nel paese dei coppoloni. Milano: Feltrinelli.

Ciabattoni, F. (2016). La citazione è sintomo d'amore. Roma: Carocci.

Ciabattoni, F. (2017). "Dante And Italy's Singer-Songwriters" in Italian Quarterly, LIV, 2II-2I4, 6I-80.

Cotugno, A. M. e Gargano, T. (2016). Dante pop. Romanzi, parodie, brand, canzoni. Bari: Progedit.

Cucco, E. (2009). Vinicio Capossela: rabdomante senza requie. Milano: Auditorium.

Fiore, T. e Capossela, V. (2006). "L'accolita dei rancorosi" in Quaderni del Novecento, a cura di Teresa Fiore, VI, I4I-I42.

Gargano, T. (2018). Dante pop. Canzoni e Cantautori. Bari: Progedit.

Isidoro di Siviglia. (2013, Etimologie. a c. di C. Leonardi. Torino: UTET.

Kristeva, J. (1980). "The Bounded Text" in Desire in Language: A Semiotic Approach to Literature and Art. Trad. Thomas Gora, Alice Jardine, e Leon S. Roudiez, a c. di L. S. Roudiez. Oxford: Blackwell.

Meisel, P. (2010). The Myth Of Popular Culture: From Dante To Dylan. Hoboken: Wiley-Blackwell.

Melville, H. (1993). Moby Dick or The Whale. Introduzione di D. Herd. Knoxville (TN): Wordsworth Classics.

Melville, H. (2009). Moby Dick o la balena. Trad. Cesare Pavese. Milano: Adelphi. (ra ed. , I94I).

Montale, E. (1984, 2000). Tutte le poesie, a c. di Giorgio Zampa, Milano: Mondadori.

Newman, L. Bertani Vozar. (1993). "Melville's Copy of Dante: Evidence of New Connections between the Commedia and Mardi" in Studies in the American Renaissance, a c. di J. Myerson. Charlottesville, University press of Virginia, 305-338.

Nuzzo, G. e Gianotti, G. F. (a c. di). (2009). I quattro libri delle Odi e l'Inno secolare 
di Quinto Orazio Flacco: tradotti in versi italiani con testo latino a fronte. Palermo: Flaccovio.

Omero. (1989). Odissea, trad. R. Calzecchi Onesti; pref. F. Codino. Torino: Einaudi. Pasquali, G. (195I). Stravaganze quarte e supreme. Venezia: Neri Pozza. (Ia ed. 1965). Salvarani, B. e Semellini, O. (2013). Dio, tu e le rose. Il tema religioso nella musica pop italiana da Nilla Pizzi a Capossela. Trento: Il margine.

Sidonio, D. (20I4). Il Portolano, n. 78/79, anno XX (luglio-dicembre 20I4), I4-I6.

Sidonio, D. (a c. di) (20I6, Mi si scusi il paragone: Canzone d'autore e letteratura da Guccini a Caparezza. Musicaos editore.

Sidonio, D. (20I6). "Vinicio Capossela. Canzoni della Cupa" I6/05/20I6 https://www. rockit.it/recensione/34959/viniciocapossela-canzoni-della-cupa.

Thomas, R. F. (2017). Why Bob Dylan Matters. Harper Collins.

\section{Discografia}

Capossela, V. (1996). Il ballo di San Vito. CGD EAST WEST.

Capossela, V. (2006). Ovunque proteggi. Atlantic Records, Warner Music Italy.

Capossela, V. (20II). Marinai, profeti e balene. La Cùpa, Warner Music Italy.

Capossela, V. (2016). Canzoni della Cupa. La Cùpa, Warner Music Italy.

Capossela, V. (2019). Ballate per uomini e bestie. La Cùpa, Warner Music Italy.

Eidel, P. (200I). Renaissance. Naïve.

Genesis. (1973). Selling England by the Pound. Charisma Records. 


\section{InTERVista-CONCERTO di Vinicio CApossela}

Trascrizione dell'intervista di Francesco Ciabattoni a Vinicio Capossela, Georgetown University, 4 Maggio 2019. Si ringraziano l'Istituto Italiano di Cultura di Washington (DC) e il suo direttore Emanuele Amendola. Si ringraziano anche il Dipartimento di Performing Arts della Georgetown University, in particolare il professor Anthony Deldonna per aver reso possibile il concerto e l'intervista. L'intervista si avvaleva della traduzione consecutiva di Ilaria Isabel Luce, a cui vanno pure i nostri ringraziamenti. Si trovano nel testo della presente intervista alcuni riferimenti agli interventi dell'interprete. Infine, un grazie anche a Angelica Rossi-Hawkins per l'aiuto con la trascrizione e gli aspetti tecnici.

Francesco: Allora sì, sì.

Vinicio: Sì.

Francesco: Siamo quasi in orario, the whole show will take about an hour, hour and a half — we are fluid. Siamo fluidi, e per cominciare, ovviamente il titolo di questo spettacolo, che è Poveri Cristi.

Vinicio: ah! Alla fine abbiamo scelto questo!

Francesco: Credo di sì! O, sennò, ovunque correggi e orienta.

Vinicio: Sì, ma l'abbiamo scelto perché da... abbiamo appena pubblicato questa canzone che si chiama "Il Povero Cristo" e abbiamo pure girato un piccolo filmino, ed è un'espressione straordinaria, no, "Povero Cristo?" Che però quando ho fatto tradurre la canzone in Inglese non corrisponde. Sì, guarda, si usa dire poor devil; poor soul; ma poor Christ non ha lo stesso significato che ha per noi. Quando si dice invece "povero Cristo," nella lingua Italiana, soprattutto in meridione, il Cristo si è fatto così uomo, si è incarnato così bene, che si è impoverito, ed è diventato la metafora, proprio, della condizione umana, no? Il 'povero Cristo' è chiunque, insomma, viva vite, viva una vita... porti la sua croce, ecco. C'era un bellissimo libro che si chiamava Cristo tra i muratori. Chi l'ha scritto?

Francesco: Poi guardiamo.

Vinicio: Uno scrittore Italo-Americano, è un libro degli anni 5o. Allora pensavo che... che forse era, era un titolo adatto...

Francesco: Ah, Christ in Concrete di Pietro di Donato?

Vinicio: Esatto

Francesco: ah, ok. Sì, sì.

Vinicio: Oh, thank you! So beautifully clear, and so beautifully English all this, this... queste piccole cose che ho provato a dire! Allora, una cosa devo dire su questo filmino, che l'abbiamo... lo trovate su YouTube... ed è stato girato a Riace. Riace è un posto simbolo, in questo momento ancora di 
più... per quanto succede nel Mediterraneo e per il periodo che sta vivendo l'Italia, insomma, questa... A Riace si è sperimentato un modello che a mio parere tentava di mettere in atto la 'buona novella,' no? Cioè, il cui principio è 'ama il prossimo tuo come te stesso,' ed è anche un modello che è stato negato perché... anche per ragioni... sì, amministrative, ma anche politiche, attualmente il modello Riace è stato smantellato. Il sindaco, Domenico Lucano, è in... è confinato nel paese di fianco, che è una cosa surreale, no? E noi abbiamo girato lì a Riace questo film, con la regia di un grande direttore della fotografia, il regista che è Daniele Ciprì, ma soprattutto avendo come interprete Enrique Irazoqui, che era l'uomo che a I9 anni nel '64 interpretò il Cristo del Vangelo Secondo Matteo di Pasolini. Quello è stato un... un bellissimo incontro.

Francesco: Parlando di Pasolini, Vinicio, mi fai venire in mente il mito e il sacro... beh, parlando di Pasolini, dopo aver letto Il paese dei coppoloni, che ha molti temi che risuonano o si incrociano con quelli de Le canzoni della Cupa, ma pure dell'ultimo album — dico 'ultimo' ma in realtà 'prossimo,' perché ancora non è uscito...

Vinicio: No, esce il is di Maggio.

Francesco: Ballate per uomini e bestie. E quale delle due ci canti stasera? Per uomini o per le bestie?

Vinicio: Uomini, bestie, e poveri Cristiani.

Francesco: E poveri Cristiani! Pensavo a Pasolini e pensavo al titolo, no? Del Paese dei coppoloni perché forse in qualche modo i Coppoloni sono i poveri Cristi? Come possiamo metterli in relazione?

Vinicio: Certo, certo, come no. Molto, molto bene. È una relazione che non avevo pensato ma che capisco ora; it's a relation that now I understand. Allora, sì, Coppoloni... perché... tutti questi fatti, c'è una profonda connessione, no? Riace, perché ha ospitato i migranti? Perché è stata svuotata, no? Ed è uno dei paesi come... gran parte dei paesi dell'interno di tutto l'Appennino, delle cosiddette "Terre dell'Osso,"... hanno subito tutte la stessa sorte già profetizzata da Pasolini negli anni '5o/'60, no? Quindi l'estinzione proprio della civiltà contadina, di quel mondo, del mondo dei mestieri, ed il mondo... anche dell'identità locale, no? Questi paesi sono stati grandemente svuotati e ora sono quasi in estinzione. E quindi da quel flusso, da quella evoluzione, da quella anche fine di questa civiltà viene anche questo libro che è un libro in questi paesi vuoti, che però risuonano del mito. Ma voglio fare anche un po' lavorare la nostra interprete

Francesco: Sì, sì, certo, che la mia mente poi fonde...

Vinicio: Ci vorranno quattro ore oggi! Prima che iniziamo anche solo a far la prima canzone!

Francesco: A che ora ce l'hai l'aereo? 
Vinicio: (ride) Eh, sì. Questa era solo l'introduzione. Ma voglio solo dire questo: sì, poveri Cristi e coppoloni è vero perché questi mettevano queste grosse "peasant hat," no? Era gente da treno, gente da lavoro, gente da migrazione, gente da macello, come dicevano loro. E questo era un po' il mondo di mio padre, pieno di mitologie locali, di mitologie paesane, che io ho cercato di trasporre usando, in maniera anche comica, a volte, il registro del canto epico. Una cosa così, no? Coppoloni! [Vinicio legge dal libro] "Gente da macello, gente all'avventura, gente senza istruzione, gente da battaglia, carne avvezza a patir che dolor non sente, gente da strapazzo, gente da lavoro, gente da portare pane onesto alla sacrosanta casa e famiglia. Non siamo i Trozzoli, i Berrilli, quelli col cappello. Quelli che ti fanno levare il cappello, quelli stanno a posti in ogni posto. Noi siamo coppoloni e basta. Ma la coppola che ci oscura la testa, pure, ci fa prendere il volo nel cielo." Non tradurlo perché... (risata)... its sound in Italian, no? No, però questa cosa delle coppole grosse era una cosa mitologica, sempre nasceva dalla presa in giro, no? Come si dice 'presa in giro'?

Ilaria: Make fun of?

Vinicio: Ah, fun of?

Vinicio: Il paese di mio padre aveva di fronte un paese che era più alto, su un cocuzzolo, no? Siccome stavano molto in alto, tirava vento, tirava molto più vento, per prenderli in giro dicevano che erano coppoloni perché avevano dei cappelli più, più grossi no? Per difendersi...

Quindi loro non sentivano niente, no? Dentro queste coppole... non capivano niente! Erano oscurati dalla loro coppola, no? Quindi erano diventati immaginari, no? Immaginavano molto! Non capivano niente, e immaginavano molto. E dicevano che sotto il loro paese, nella rupe, i senni dell'intelletto (e qua c'è anche Ariosto, eh?) si sviluppavano, crescevano in forma di mosconi, no? Quindi la rupe ronzava di mosconi e loro, e loro diventavano... diventavano dotti, diventavano immaginifici, avevano la conoscenza, ecco. La canoscenza, come la chiama Dante. E gli arrivava per questi mosconi. Ecco. E finisco con i coppoloni per dire quanto erano proprio, illuminati, che una volta - questo paese è veramente sopra un cocuzzolo - una volta arrivò una grande nebbia dalla pianura sotto, e loro pensavano che era arrivato il mare, e si misero... tutto il paese si buttò giù dalla rupe con delle barche fatte con le fazzatore, che erano delle cose dove, quando facevano... scannavano il maiale, lì dentro raccoglievano il sangue. Quindi erano delle specie di canoe che usavano per far la pasta, insomma, no? E quindi con queste [suono onomatopeico], e per questo non c'è neanche più un Cairanese. Sono tutti emigrati in America! Esatto, esatto. Quindi, tutte queste fesserie sono raccontate in questo libro, però usando il canto epico, cioè il metro dell'Odissea, dell'Iliade, qualche volta 
anche di Dante, insomma, c'è un lavoro sulla lingua per cercare di passare dal dialetto, che è irriproducibile sul... sul... tradurlo in canto epico, ecco.

Francesco: Infatti è proprio il registro epico, che pervade alcune delle tue canzoni. Magari non tutte, ma alcune, e anche certe pagine dei tuoi libri. $\mathrm{Mi}$ fa pensare al mito, come dicevamo, il mito locale che diventa universale. E ciò si collega alla dimensione del sacro. Vinicio Capossela, cantautore del sacro, e anche scrittore del sacro. Mi pare che tu sia alla ricerca delle tante forme diverse del sacro che affondano le radici in antichi miti e leggende.

Vinicio: Mi interessa la ritualità. Mi interessa la ritualità anche perché è stata completamente soppiantata, sostituita dalla procedura. Per esempio, per venire in America c'è una grande procedura, no?

Francesco: Come no!

Vinicio: E quindi... no complimenti, è proprio la procedura, no? Uno poteva, un tempo c'era la ritualità, no? La fazzatora, è arrivata la nebbia, è il momento di andare in America... no! Adesso c'è la procedura. E questo è un cambiamento culturale, no?

Francesco: Certo. Sì.

Vinicio: Allora, questo disco, Ballate per nomini e bestie, attinge molto ai simbolismi del Medioevo, ai bestiari medievali. Soprattutto perché nel medioevo il reale, l'oggettivo, non coincideva col vero. Il vero era metafisico, inconoscibile. Allora, io credo che l'unico valore che può avere l'esperienza poetica è quello di riportare questo grado di verità, cioè qualcosa che è proprio il contrario della realtà oggettiva, no? E quindi, anche sostituire alla procedura il senso del sacro, della ritualità. Infatti, anche alla Casa Bianca ci deve essere una procedura però manca la ritualità!

Francesco: Assolutamente!

Vinicio: E poi...se non c'è nessuno della Casa Bianca qua dentro!

Francesco: Non vi faremo nulla, non vi preoccupate! Ma è una perfetta scelta lessicale: la procedura come opposto della ritualità, perché la procedura è costituita dai medesimi atti senza però il sacro, senza anima, senza significato. E a proposito del sacri, tu parlavi di Dante. Mi chiedo spesso perché, quando uno scrive romanzi o canzoni che parlano dell'oggi, che sono ancorate all'attualità, vuole tuttavia tornare indietro ai classici. Perché Dante?

Vinicio: però non direi "tornare indietro" ma "andare avanti!"

Francesco: Giusto.

Vinicio: Salire di piano, no? È come prendere l'ascensore e da lì uno vede tutto, non sta scendendo nella cantina.

Francesco: "Stairway to heaven", I guess.

Vinicio: Sì, certo. Forse a quello pensavano. Però tornando al sacro, è un fatto vero che la cultura popolare ha sempre avuto il senso del sacro, no? E questo è un lavoro sulla cultura popolare. Allo stesso tempo, la letteratura c'ha 
riportato questo, ha sacralizzato il mondo. Per cui c'è per esempio Dante, che ci da una visione dell'uomo in relazione al sacro ed è una cosa straordinaria, sempre più straordinaria, in un mondo disabitato dal sacro. E quando il mondo è disabitato dal sacro, allora interviene... ecco, quando il mondo è abbandonato dal sacro, interviene Dan Brown.

Francesco: So, you've introduced us to hell, I believe. Just kidding. Ma no, io sono d'accordo certamente.

Vinicio: Vogliamo fare un esempio dell'attualità di Dante? Allora, pensiamo al Mediterraneo, no? Pensiamo a questa, a questo meraviglioso Canto ventiseiesimo, dove Dante descrive proprio questo discorso enorme che fa Ulisse, "fatti non foste a viver come bruti," e si passa. Ecco, pensate che differenza-lì non c'è la procedura! Lì c'è proprio l'andare oltre un limite! Il fatto proprio di andare alla conoscenza, ma infrangere anche un totale equilibrio, per cui si finisce per aver superato un limite, si finisce per essere sommersi dal mare. Secondo me c'è già tutto in questo canto ventiseiesimo e pensate all'attualità di quel canto, cioè quanti in nome della conoscenza, della sopravvivenza, tutto quanto, io penso che veramente ognuno che oggi cerca di attraversare il Mediterraneo è un nuovo Ulisse! Un Ulisse esattamente come lo descrive Dante.

Francesco: A volte penso che se l'Ulisse dantesco non fosse morto in mare avrebbe continuato la navigazione verso ovest, "di retro al sol," e sarebbe finito nelle maglie della Homeland Security qui ... che forse sarebbe stato comunque un inferno per lui. Scherzo, ovviamente, ma forse lo è per qualcuno.

Vinicio: No, però è vero! Io ho scelto quella canzone anche perché porta direttamente in America!

Francesco: Si, già, infatti. Se tutto va bene!

Vinicio: Questo era proprio il limite che non bisognava varcare! E allora...

Francesco: Cosa ci canti?

Vinicio: Bene, come a Sanremo dicono, una canzone molto pop, vedrete, si chiama "Nostos."

Francesco: Fantastico! Noi cosa facciamo?

Vinicio: Rimanete qua, perché poi...

Francesco: Balliamo?

Vinicio: Non c'è da ballare! Non c'è da... c'è solo proprio da... da...

Francesco: Meglio, sono più a mio agio!

Vinicio: Niente. Questo brano, sentirete, non è molto... non è una canzone fatta di strofa, ritornello, è un... è una... ho provato diverse volte a mettere in musica delle, delle pagine di letteratura. Questa è una citazione di questo... di questo passaggio, ecco. "Nostos" perché? Perché "Nostos" è proprio la categoria, no, del... Lei, parli lei del Nostos, no? Lei che è... 
Francesco: Il nostos è il ritorno, e insieme il desiderio del ritorno, diventa un tema letterario è da qui che viene la parola nostalgia e Ulisse-almeno quello di Omero-è un po' l'emblema del desiderio di tornare, ma ironicamente l'Ulisse di Dante fa piuttosto il contrario : diviene l'emblema di una sete di conoscenza che lo spinge a ripartire per cercare di accumulare altra conoscenza, anche se è già vecchio. Ulisse non segue la procedura.

Vinicio: E ora ho il piacere di invitare sul palco un clandestino appena arrivato dal Messico, ha avuto la sua Odissea per arrivare qui... dal confine sud.

Francesco: He's not a real coppolone, but ok.

[Vinicio esegue "Nostos"]

Vinicio: Ecco, questo era il passaggio che dicevamo. Ora, ora vorrei anche fare un altro brano. Si chiama "La Lancia del Pelide" perché nel mito niente è mai affidato al caso. C'è un episodio che ricorda proprio Dante, dice così, "una medesma lingua pria mi morse, sì che mi tinse l'una e l'altra guancia, e poi la medicina mi riporse. Così od'io che solea far la lancia d'Achille e del suo padre esser cagione prima di trista e poi di buona mancia." Questo fatto che la, quella lancia che è stata fabbricata dal padre di Achille ha....ha una caratteristica che, se ti colpisce, ti uccide, ma la stessa lancia con un secondo colpo può guarire, è la sola cosa che può guarire la ferita che ha inflitto. È dunque come gli occhi dell'amata.

[Vinicio esegue "Lancia del Pelide"]

Vinicio: Bene. Allora, rimanendo nel dipartimento medioevale, ora facciamo una canzone ispirata alla danza macabra.

[Vinicio esegue "Danza Macabra”]

Vinicio: E così dopo Dan Brown, Tim Burton! Beh, la danza Macabra, diciamo, era un piccolo dono per il nostro professore di dantistica. E ora possiamo andare avanti nel medioevo. Vi volevo proporre questo esercizio. $\mathrm{O}$ avete delle domande?

Francesco: questa è dal nuovo album vero?

Vinicio: sì, il vecchio nuovo album.

Francesco: Benissimo, non interrompiamo il flusso, vai pure, qualunque cosa tu voglia fare, noi ti seguiamo. Visto che ci siamo, la danza macabra, le Livre Vermeil, c'è l'idea della morte che ci insegue. E' questo uno dei temi principali del tuo nuovo album?

Vinicio: Sì, beh, ci sono delle categorie che appartengono al medioevo, no? La 
danza macabra... perché sono adatte a questo periodo di post-medioevo. Per esempio, la danza macabra è molto contemporanea perché, a cosa serviva la danza macabra? A mettere paura, no? A mettere paura, e quindi era una cosa che serviva molto al potere. Ho una mia teoria personale. Nella cultura popolare c'è lo spavento. Lo spavento è educativo. Uno si educa a superare la paura, mentre invece la paura è paralizzante. E c'è un lavoro massiccio sulla paura, sulla paralisi della paura, e questo naturalmente a fini di gestione del potere. [Dopo la traduzione di Ilaria il pubblico mostra cenni di assenso]. Adesso siete d'accordo, eh? si, c'è un lavoro molto... (sottovoce: aspetta, ora c'è da fare un duetto).

Vinicio: Yes. I would like propose you this... Ballad of Reading Gaol. È una canzone che è stata tratta da questo meraviglioso testo finale di Oscar Wilde. Oscar Wilde ha avuto una... Oscar Wilde ha avuto un periodo in cui si veniva a guadagnare da vivere facendo lezioni meravigliose in America! Avrei voluto essere uno studente. Ma è finito... ha avuto una delle cadute più straordinarie della storia, e in questa parte finale della sua vita scopre dall'estetismo, dalla cosa, insomma, proprio, la compassione. E questo ultimo suo componimento è proprio la ballata del carcere di Reading, che trovo esemplare proprio per questa denuncia, intanto, del tradimento del messaggio Cristiano, la denuncia della pena di morte, la denuncia dell'inumanità del carcere. Ha delle pagine sul Cristo come poeta che sono meravigliose. Allora io propongo questo duetto. Questo è l'originale, partiamo dalla prima.

[Ilaria legge The Ballad of Reading Gaol di Oscar Wilde, quindi Vinicio esegue "Ballata del carcere di Reading"]

Vinicio: Da questo Cristo a un altro, il nostro percorso di poveri Cristi arriva a questa canzone.

[Ilaria legge la traduzione e Vinicio canta]

Vinicio: Voglio segnalare la bella mostra sulla immigrazione e sulle immigrazioni di Pietro Ruffo che è qui all'Istituto Italiano di Cultura di Washington DC. Voglio segnalare la capacità di tradurre all'istante della signora Ilaria Luce.

Francesco: Bene, vuoi fare ancora un'ultima cosa?

Vinicio: Sì, voglio fare un'ultima cosa così ci lasciamo in maniera un po' più, diciamo....

Francesco: Sì! Uplifting!

Vinicio: Sì, sennò...poveri Cristi sì, ma il povero Cristo, comunque, quand'è 
tempo di... Questo è un pezzo da Canzoni della Cupa e... it's a rural, rural... un album rurale, ecco. Ci sono un po' di canzoni che ho estratto dai sonetti che si usa cantare a Calitri, che è questo piccolo paese dell'alta Irpinia, this little village of Irpinia, where my father is from. Questo per esempio lo sentite cantare da una vecchia signora, old woman, parlava dell'amore di questa, di questa ragazza che si chiama Teodora, per il suo giovane marito che sta morendo. Arriva una commara e gli dice, "Basta piangere! Se muore Liberto, ti pigli a Vituccio!" E lei però gli dice, "None, none, none! È per Liberto la passione!"

Francesco: Questa, decisamente, è una canzone che mostra le radici folk della tua musica.

Vinicio: Eh, folk roots, yes.

Francesco: Grazie, Vinicio.

Vinicio: Abbiamo finito, o dobbiamo continuare?

Francesco: Come vuoi tu! Io sto anche fino a domani, non è un problema.

Vinicio: Alcohol free?

Francesco: Alcohol free qui dentro, però fuori no.

Vinicio: Ma neanche con un sacchettino? Io...

Francesco: Ecco, forse 'sta cosa del sacchettino sta a fagiolo, infatti. Quindi?

Vinicio: Questa qua è una canzone di Matteo Salvatore, un grande povero Cristo, che ha scritto, in campo di poveri cristi, il verso sullo sfruttamento del lavoro più straordinario mai scritto: Padrone mio, ti voglio arricchire. Questa invece è una canzone d'amore.

Francesco: Grazie a Vinicio! Noi vi ringraziamo, ringraziamo Vinicio e tutta l'organizzazione, domani, credo di nuovo, al Millennium Stage, con tutta la band! La band full steam. Che succederà domani, Vinicio?

Vinicio: Non saremo molti di più, la band al completo è di tre persone. Domani sarà un po' più breve. Anzi siccome domani non potremo stare più di un'ora, stasera vi canto ancora una canzone! Questa è ispirata a Winesburg Ohio, di Sherwood Anderson, un libro meraviglioso che questa America, alla fine della civiltà contadina e all'alba della civiltà industriale, dove tutti hanno nomi presi dalla bibbia, grandi solitudini, che mi ricordano il piccolo mondo di Scandiano, da dove c'è anche una mia compagna qua...

[Vinicio esegue "Poveri Cristi"]

Vinicio: Era un piccolo omaggio, grazie, thank you so much, grazie a tutti! Francesco: Grazie mille! 